\title{
Progress of Resources and Environmental Carrying Capacity
}

\author{
Y. N. Tian and H. Q. Wang
}

\begin{abstract}
Resources and environmental carrying capacity as the basis of sustain development has been paid more and more attention. This paper aims to discuss the major concepts of resources and environmental carrying capacity and the problem in this area. Based on thorough analysis of the concept and evaluation methods of resources and environmental carrying capacity, it has been concluded that the conceptual model deserves more attention, and the development direction of resources and environmental carrying capacity has been pointed out.
\end{abstract}

Index Terms-Resources and environmental carrying capacity, assess method, conceptual models

\section{INTRODUCTION}

China is a developing country with a large population, which has the unprecedented growth and progress in decades since reform and opening-up. Development improves people's living conditions, at the same time, gradually challenges to our survival environment. How to achieve sustainable development, and avoid the limits of resources and environment became one of the important things in China and even in world. Resources and environmental carrying capacity seems to be the promising method to solve these problems. It aims at coordinating the relationship between the social economic activities and environment, so that it is the best tool to realize regional sustainable development and becomes regional planning foundation. In recent years, this concept has been used in many places, and numerous access methods appear in the meanwhile. However, there is still no agreement in the concept and access methods and little discussion has yet been given to this problem. The process of the concept and the access methods have been summarized in this paper so that it may facilitate the research of resources and environmental carrying capacity in the future.

\section{EVOLUTION OF CONCEPT OF RESOURCES AND ENVIRONMENTAL CARRYING CAPACITY}

The term carrying capacity stems from the mechanical, and borrowed by theoretical genecology firstly. Originally it means the maximum number of specific species in a biota that could be maintained by the biota's various resources [1], [2]. It provided guidance for rang management in this period.

Manuscript received November 25, 2012; revised January 17, 2013. This work was supported by Ministry of Land and Resources of the People's Republic of China under Grant 1212011220097.

The authors are with College of Water Sciences, Beijing Normal University, 19 Xinjiekouwai Street, Beijing, China (e-mail: tyn229@yahoo.com.cn; whongqi@126.com).
With the global population continues to increase, farmland area decreases and the food crisis, the concept of carrying capacity are gradually changed and used to solve the problems appeared during the development of human society. The carrying capacity of resources was gradually noticed first due to the land resources shortage. The accelerated process of industrialization made the research of single factor resource carrying capacity gradually extended to the water resources, mineral resources and forest resources because it started to have problem in these areas. In 1980s, UNESCO gave the concept of resources carrying capacity which made all kinds of resources to be considered as a union, and used population size as the expression of resources carrying capacity [3]. It preliminary formed the basic framework of the concept of carrying capacity.

The unreasonable utilization of resources let to environmental degradation and environment pollution. This phenomenon, on one hand, decreased the utilization value of resource, thus resource shortage aggravated. On the other hand, the pollution of air, water and soil threatened to human survival. In the face of this dilemma, people began to think how much pollution can environment take, and how much strength of human activities can earth afford, so environmental carrying capacity arose to solve these questions. In the 1970s, Godschalk and Parker explored the use of three types of environmental, institutional, and perceptual carrying capacity in natural and man-made systems [4]. Subsequently, environmental carrying capacity became a valuable tool for environmental planning and management [5]. In 1996, the Florida Keys Carrying Capacity Study, conducted by the State of Florida and the US Army Corps of Engineers, tried to convert the vague carrying capacity concept into a functional, quantitative method. [6]. In 2002, the United States Environmental Protection Agency (EPA) evaluated environmental carrying capacity of the 4 lakes, and gave the suggestion about water quality protection of the lakes. The concept of environmental carrying capacity proposed strictly in China was in the planning of Meizhou Bay which been defined as in a certain period of time and a certain area, as well as a certain natural environmental condition, to keep the structure and function of environmental not change and failure, environment system can afford the threshold of human activities [7]. Environmental carrying capacity gave the guidance to the protection of environment, and both the theory and the method contribute to the later researches.

As the deepening of environment cognition, it has been realized that ecological environment should be treated as a whole, and single factor evaluation of carrying capacity could not meet the requirements of sustainable development. Resources and environmental carrying capacity as a 
comprehensive concept generated in order to solve the complex problem. This concept not only considered various impact factors of resource and environment but also the social and economic factors. The definition of this concept paid attention to overall effect but it combined the single factor simply at the first beginning. Hong et al.[8] gave the definition of the resources and environment carrying capacity that is under the condition of rational exploitation of resources and ecological environment health, the ability of resources and environmental system in different scale areas that it could afford the population size and the amount of social and economic gross in a certain period of time. The concept of environmental carrying capacity, ecological carrying capacity and the regional carrying capacity has evolved into the comprehensive definition at the same time. Zhu et al. [9] defined the eco-environmental carrying capacity related to water as the largest population and economic scale (usually expressed as gross domestic product, GDP) that can be sustained in a particular region, over a specified time, under a certain environmental protection standard and social welfare level, when the eco-environments, society and economy of the region reach a critical acceptable state by using the local (or diverted from outside) water and other resources available. Liu and Borthwick [10] proposed a comprehensive measurement system for environmental carrying capacity which comprises models of natural resources capacity, environmental assimilative capacity, ecosystem services capacity and society supporting capacity, and applied this measurement system to a case study of Ningbo, China.

All above shows that the concept of carrying capacity develop from the ecological carrying capacity, to single impact factor carrying capacity, to resources and environmental carrying capacity. The research preferred to consider single resources or environmental impact factor initially, and then it has been realized that to solve the complex problems, the resources and environmental system should be treated as a whole. In order to achieve this goal, the method of comprehensive evaluations has been explored, and the connotation and extension of resources and environmental carrying capacity still is the focus. In the last decade, the human social and economic activities are gradually incorporated into the carrying capacity research category so that the concept of resources and environmental carrying capacity has become an aggregate concept eventually.

Although the carrying capacity seem to be an obvious concept to describe the relationship between human society and nature environment, but what it exactly mean still is bone of contention, and different scholar has different understanding. In addition to this, the evaluation of it involves lots of content including conceptual model construction, evaluation index screening, evaluation index weight distribution, classification, evaluation method established and the corresponding prediction analysis model, all of which should be considered seriously. Due to many evaluation steps, together with different understanding of the concept, many evaluation theory and method has been presented, which is badly in need of being sorted. This paper aims at summarizing more applied theories and methods in order to finding development direction of research. Meanwhile, the understanding of the concept is also argued based on many studies before.

\section{DEVELOPMENT TREND}

\section{A. Analysis of Conception}

Carrying capacity has involved in many research fields such as environmental carrying capacity, ecological carrying capacity, regional carrying capacity and resources and environmental carrying capacity. All of these are comprehensive carrying capacity, except a slight difference. Environmental carrying capacity and resources carrying capacity is almost the same, both of which consider not only one single factor. Environmental carrying capacity actually refers to the generalized environment, which contains both of the resources and environmental factors. However, it is not clear expression, and more concern about environment will be paid in many conditions. Resources carrying capacity has the same problems. Resources and environmental carrying capacity values the resources as highly as the environment, both of which have a giant effect on human society, so that it contributes to understanding of the complexity of ecosystem in depth. Ecological carrying capacity emphasizes the stable and health of ecological processes, species, communities, and the population, while resources and environmental carrying capacity aims at evolution which benefits the mankind. Ecological carrying capacity is a complex concept, but it focus on the state of nature and protecting others rather than human. Regional Carrying Capacity put the emphasis on the scale. Essence of resources and environmental carrying capacity is to reveal the coordinate degree between human and nature, so the most important thing is to indicate the state of resources and environmental system under human intervention and find the key factor of resources and environment which constrain the development. It can be seen that this concept is especially suitable for the conditions with large people and resources and environment scare in China.

Although the concept still needs to be discussed, the main content can make sure. Firstly, certain period and certain region contain in the concept of resources and environmental carrying capacity should be mentioned, because it reflects not only scale effect in time and space., but also emphasis on the sustainable development, which means the precondition of carrying capacity must protect original environment against deteriorate, or even could turn into the direction that benefit to human through regulating action of human. Secondly, the pattern and the level of social economic development influence resource and environment differently, so it is one of the most important focuses as well as the object of adjusting. Under the same development levels, population size and economic scale have the same dependencies upon the resource and environment. Thirdly, the goal of carrying capacity evaluation is to protect eco-environment and guarantee rational utilization of resources. Finally, the carried objects of carrying capacity are population size and social economic scale, which is crucial importance to people survival and development. Therefore, evaluation of resources and environmental carrying capacity should analyze the stage 
of economic development first.

Theoretical studies support the practical application. The research about the theory of resources and environmental carrying capacity deeply and systematical has important guiding significance to the evaluation. Therefore, the theory study must be focused.

\section{B. Index of Evaluation}

Resources and environmental system is a complex system, thus it has the characteristics of self-organization, openness, hierarchical organization and scale effect. Resources and environment carrying capacity study only embodies all of these characteristics it maybe response real situation. Ignorance of scale effect in the study of resources and environmental carrying capacity is one of the most important reasons for difficult to application. The index system is not only able to represent all elements which influence resources and environmental carrying capacity, but it also reflects the scale effect in different environmental system. Although, the index system is such important, the method of constructing is rare which need more explore.

In addition, the current resources and environment carrying capacity study are mostly static research, and lack of consideration about the openness. This is another reason which causes the usage difficult. With the economic development, the exchanges between different places are more and more. If the openness is not considered, the evaluation of carrying capacity will overvalued or underrate, both of which is not expected. For example, a city system is open more, and it depends on input from outside to maintain the development. If the carrying capacity were overvalued, the system will take more risk; if instead will be doubt the theory which is why there are some researcher suspect the carrying capacity. So the openness should be considered more in the index of evaluation.

\section{Evaluation Method}

The evaluation of resources and environmental carrying capacity involves many contents including conceptual model, evaluation index, evaluation, weight of indicator, and forecast model. In addition, there are many different understanding about the concept of carrying capacity, so that it appears many evaluation theory and method. In this paper methods used more were summarized.

The single element carrying capacity evaluation method started early, so the theoretical system and calculation method is relatively mature, and it provides necessary foundation to the evaluation. This method calculate different kind of resources and environmental evaluation respectively, and then summed them up to obtain the resources and environmental carrying capacity, as in (1).

$$
I=\sum_{i=1}^{n} \omega_{i} \bullet I_{i}
$$

where $\omega_{i}$ is the weight of the indicator $i, I_{i}$ is the $i^{\text {th }}$ indicator, $\mathrm{I}$ is resources and environmental carrying capacity.

The theory of complex system emphasize on that the whole is greater than the sum of part, and this method cannot solve this problem. That is why multi-objective decision analysis method appeared. The overall thinking of this method is that several elements such as population, environment, and resources and so on should be chosen first as the goals. Through the multipurpose weigh determines carrying capacity in different social development mode, different economic structure, different resources usage and different background of environment. Based on the analysis of the relationship between different elements, system state is described with mathematical programming, and to make sure multi-object optimization. Multi objective analysis decision analysis method does not pursue the single object optimization, only the pursuit of the overall optimal solution. This solved the problem existence in the single factor evaluation method, and fully reflects the status of resource carrying capacity.

The state space method based on the Euclidean geometry space is an effective method to quantitatively describe the state of the system. It consists of n-dimensional state space each of which represents an element of the system. This is an important method of quantitative description and measurement of carrying capacity. Although the state space method is a quantitative calculation method, and the construction of carrying capacity surface is difficult as well as lots of information are required, it can still accurately judge carrying capacity in an area within a certain time, meanwhile it can make sure the result is reasonable.

The method based on model can be traced back to the late 1960s to early 1970s. Slesser [11] proposed "ECCO" model as a new model of resources and environmental carrying capacity calculation method. Under the assumption that "everything is energy", this method considered the correlation between population, resources, environment and development, then establishes the system dynamics model which take energy as conversion standard to simulate different development strategy. It simulated the relationship between population and carrying capacity under the different strategy so that it could explore the optimization which was benefit to the sustain development. This method could explain the relationship between the structure and function of complex system, is good at deal with higher-order wave problem and quantitative analysis different characteristics of the systems so that it is suitable to describe the trend. With the more and more recognition of resources and environmental system, this method has been used more and more, and different models continue to spring up such as cellular automata (CA) and multi-agent applications which show the great prospects.

The theory of in spite of one of the basis of carrying capacity, they are still some difference. Carrying capacity aims to emphasize on carrying the population, social and economic scale. However, the ecological footprint is to act in a diametrically opposite way. In one hand, it tries to calculate bio-capacity in the area as the standard of sustain development; on the other hand, it ties to estimate actual carrying capacity the area consumed, namely the calculation of ecological footprint.

\section{Conclusion}

Carrying capacity study experienced a century of history, but has not been able to solve the practical application of how 
to convert to the practical application from abstract concepts. Therefore, the most important step for the carrying capacity is the embodiment of the concept. So far, the evaluation method on how to characterize the results of carrying capacity is very inadequate, thus looking for the evaluation method for the study of the carrying capacity concept, clearing calculation process and the expression of carrying capacity all are very important. Although the scale was mentioned in the definition of carrying capacity, in the actual evaluation process rarely involved this issue, which should be concerned in future. In conclusion, the study of resources and environmental carrying capacity is an important exploration of the human endeavor to live in harmony with nature, and an important foundation to achieve sustainable. With human understanding about nature improving, the research about resources and environmental carrying capacity study will be bound to play a greater significance.

\section{REFERENCES}

[1] Y. D. Lin and M. H. Ying, "Regional carrying capacity: case studies of Bohai Rim area," Journal of Geographical Sciences, vol. 12, pp. $177-185,2002$.

[2] D. Price, "Carrying capacity reconsidered," Population \& Environment, vol. 21, pp. 5-26, 1999.

[3] I. A. S. Hadwen and L. J. Palmer, Reindeer in Alaska: US Department of Agriculture, 1922.

[4] D. R. Godschalk and F. H. Parker, "Carrying capacity: a key to environmental planning," Journal of Soil and Water Conservation, vol. 30, pp. 160-165, 1975.

[5] J. H. Baldwin, Environmental planning and management, Westview Press Boulder, BC, Canada, 1985.
[6] A. L. Clarke, "Assessing the carrying capacity of the Florida Keys," Population and Environment, vol. 23, pp. 405-418, Mar, 2002.

[7] W. Zeng, et al., "Theory of environment carrying capacity and it's application of environmetal planning on Meizhou Bay," China Environmental Science, vol. 18, pp. 70-73, 1998.

[8] Y. Hong and W. Ye, "Theoretical Analysis of Sustainable Environmental Carrying Capacity," China Population, Resources and Environment, vol. 8, pp. 54-58, 1998 .

[9] Y. Zhu, et al., "Analysis of Temporal and Spatial Differences in Eco-environmental Carrying Capacity Related to Water in the Haihe River Basins, China," Water Resources Management, vol. 24, pp. 1089-1105, April, 2010.

[10] R. Z. Liu and A. G. L. Borthwick, "Measurement and assessment of carrying capacity of the environment in Ningbo, China," Journal of Environmental Management, vol. 92, pp. 2047-2053, Aug, 2011.

[11] M. Sleeser, "Enhancement of carrying capacity options ECCO," The Resource Use Institute, vol. 10, pp. 5, 1990.

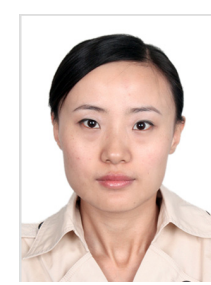

Y. N. Tian was born in Tangshan city, Hebei province, China in 29 February 1984. The B.S. degree in environmental engineering was received from the North University of China at Taiyuan city, the M.S degree in environmental science from the Beijing Normal University in 2007 and 2010, respectively. She is studying for a doctor's degree in Beijing Normal University.

She has been engaged at least 3 years in the research about resources and environmental carrying capacity. She participated in the program named "The indicators of resources and environmental carrying capacity in different scale" and "The indicators of resources and environmental carrying capacity in ecological areas", both of which is the basis of territorial planning in China. 